\title{
QuiRA: An Augmented Reality Application to Support Chemistry Learning
}

\author{
Letícia Porto Soares \\ Instituto Federal da Bahia \\ Vitória da Conquista, Brasil \\ leticiaportosoares@gmail.com
}

\author{
Manuela Amaral de Araújo \\ Instituto Federal da Bahia \\ Vitória da Conquista, Brasil \\ mn21922192@gmail.com
}

\author{
Camilo Alves Carvalho \\ Instituto Federal da Bahia \\ Vitória da Conquista, Brasil \\ camilocarvalho@ifba.edu.br
}

\author{
Paulo Henrique Teixeira Silva \\ Instituto Federal da Bahia \\ Vitória da Conquista, Brasil \\ paulohenrique-201119@hotmail.com
}

\author{
Luis Paulo da Silva Carvalho \\ Instituto Federal da Bahia \\ Vitória da Conquista, Brasil \\ luispscarvalho@gmail.com
}

\author{
Wdson Costa Santos \\ Instituto Federal da Bahia \\ Vitória da Conquista, Brasil \\ wdsoncosta@ifba.edu.br
}

\begin{abstract}
The volume of information which contemporary student has access is huge, and they are surrounded by smartphones, tablets, internet and computers in an almost inseparable way from their daily life. Therefore, we think that it is important that schools should consider the insertion of technological instruments in the classroom to attend the students' interest in these devices. One of the alternatives is combining Augmented Reality and mobile technology to enable the interaction with virtual objects by overlapping them with real environment. These technologies may become allied in the process of teaching and learning abstract concepts of various subjects. In this context, QuiRA is proposed to help the teaching of Chemistry, an ideal field to explore questions about the use of computer animation due to the difficulty of visualizing its concepts in two dimensions. This article discusses the details about the QuiRA application and the demonstration of its use for visualizing chemical molecules.
\end{abstract}

\section{KEYWORDS}

Augmented Reality, ARToolKit. Chemistry.

\section{INTRODUÇÃO}

A natureza do conhecimento está em constante evolução. No entanto, os processos de ensino e aprendizagem contemporâneos são considerados obsoletos. Hoje, o volume de informações é imenso e o aluno se vê envolto por tecnologias como: smartphones, tablets, internet, computadores, entre outros, e como estão muito presentes, quase que indissociáveis ao cotidiano deles, fornecem experiências mais atrativas. De acordo com [1], cabe, então, aos educadores utilizarem instrumentos tecnológicos em sala de aula, para atender não apenas um interesse exclusivamente pedagógico, mas os interesses dos alunos.

In: XVII Workshop de Ferramentas e Aplicações (WFA 2018), Salvador, Brasil. Anais do XXIV Simpósio Brasileiro de Sistemas Multimídia e Web: Workshops e Pôsteres. Porto Alegre: Sociedade Brasileira de Computação, 2018.

(C) 2018 SBC - Sociedade Brasileira de Computação.

ISBN: 978-85-7669-435-9.
Uma das alternativas que utiliza da tecnologia para uma aprendizagem mais lúdica e efetiva é a inserção da Realidade Aumentada (RA) que, segundo [2], se caracteriza por combinar real com virtual, possuir interatividade em tempo real e ser registrado em três dimensões. A RA se trata de uma tecnologia onde os alunos poderão interagir com objetos virtuais através da sobreposição de camadas virtuais a um ambiente real [3]. Essa interação de ambientes virtuais no mundo real torna a percepção mais atraente e estimula o processo investigativo dos alunos. Considerando os avanços da tecnologia móvel e a popularização dos smartphones é possível integrar a Realidade Aumentada (RA) aos dispositivos móveis inserindo um novo paradigma à relação aluno-professor, onde a utilização desses dispositivos se torna aliada, e não um incômodo, no processo de ensino e aprendizagem de diversas disciplinas, como, química, matemática, biologia, geografia e história.

O QuiRA foi proposto para auxiliar o ensino de Química por meio da Realidade Aumentada aliada aos dispositivos móveis. O objetivo da aplicação foi direcionado para a Química, porque: (i) é um componente curricular obrigatório no ensino básico, (ii) é considerada de difícil compreensão pelos alunos e, (iii) por muitas vezes, os alunos não conseguem associar o conteúdo da disciplina ao seu dia-a-dia [4] e (iv) pela visualização em 2D não ser suficiente para a devida observação do arranjo tridimensional dos átomos nos compostos químicos [5], sendo, portanto, um conteúdo propício para explorar as potencialidades da Realidade Aumentada.

Portanto, esse trabalho tem o objetivo de trazer uma ferramenta de Realidade Aumentada, que no âmbito do ensino, proporcione aos educadores uma maneira agradável de transmitir os conteúdos aos alunos, a fim de estimular a aprendizagem. Além disso, no âmbito técnico, pretende-se apresentar uma abordagem de utilização da biblioteca de Realidade Aumentada ARToolKit em dispositivos móveis Android que dê ao usuário a possibilidade de escolher quais objetos tridimensionais ele deseja visualizar através da RA e armazená-los em um banco de dados. Este fato potencializa e colabora com a possibilidade de criação de um visualizador de RA genérico com uma biblioteca de acesso gratuito. 


\section{TRABALHOS RELACIONADOS}

Em [6], Cunha e Guimarães criaram uma ferramenta gráfica que tem o objetivo de tornar o desenvolvimento de aplicações de RA mais fácil e transparente ao usuário, neste caso professores e alunos. No seu desenvolvido foi utilizado o software Microsoft Visual Basic $6.0 \mathrm{com}$ a biblioteca ARToolKit ${ }^{1}$. A ferramenta permite aos usuários a associação dos objetos virtuais aos marcadores predefinidos, não sendo permitida a inserção de novos marcadores. O seu funcionamento se dá através da sua execução em um computador de mesa com uma webcam para detectar o marcador e permitir a visualização do objeto virtual projetado.

O Serviço Nacional de Aprendizagem Industrial (SENAI) produziu aplicativos móveis que utilizam realidade aumentada no programa SENAI de Tecnologias Educacionais [7]. Com os aplicativos, os alunos podem utilizar a câmera dos dispositivos móveis, do celular ou do tablet, para reconhecimento em 3D especificamente das imagens presentes nos livros didáticos do SENAI. Além disso, os aplicativos permitem aos estudantes acessarem simuladores e vídeos, pelos quais os objetos impressos ganham movimento, imagem, som e interação.

Em [8] foi efetuado um experimento com o desenho e construção de objetos de aprendizagem baseados em realidade aumentada, voltado para alunos do ensino secundário de uma escola na Colômbia. Na ferramenta de RA foram utilizados os softwares Unity, Vuforia e Android Studio para a implementação e o 3ds Max para construção de 35 modelos em 3D. Foram produzidas imagens em duas dimensões para serem referência para os 35 modelos tridimensionais construídos. Após o estudo, foram obtidas avaliações positivas em termos: (a) de satisfação de uso; (b) com as características estéticas, funcionais e de conteúdo dos objetos; (c) e com realidade aumentada dentro das salas de aula como motivação para aprender. Os professores da escola, inclusive, destacaram a capacidade de foco dos alunos nas atividades de interação com o software.

Em [9] foi produzida a ferramenta Reações Químicas Aumentadas (Augmented Chemical Reactions) que visa auxiliar tanto alunos quanto cientistas mostrando moléculas químicas renderizadas em 3D quando um marcador é direcionado a câmera do computador. Além disso retrata a alteração dinâmica de moléculas quando se aproximam. Neste caso, usuários podem ter uma visão melhorada sobre certos comportamentos e interações entre moléculas. Para o seu desenvolvimento foram utilizados ARToolKit e UbiTrack. A ferramenta aborda duas formas de utilização. A primeira voltada aos alunos, onde a configuração é feita previamente e estes só irão utiliza-la para visualização das moléculas. E a segunda, onde, num modo especialista, o usuário irá definir as moléculas e atribuí-las a marcadores dinamicamente.

O QuiRA traz uma abordagem dinâmica no que se diz respeito à possibilidade de inclusão, pelo usuário, de novos objetos tridimensionais a aplicação, o que o diferencia dos trabalhos já existentes. As ferramentas disponíveis mais atuais

\footnotetext{
${ }^{1}$ https://github.com/artoolkit
}

proporcionam um conteúdo estático de marcadores e/ou objetos tridimensionais. Já as aplicações que permitem que sejam adicionados novos objetos 3D e associá-los a marcadores foram desenvolvidas para computadores de mesa munidos de um a webcam. Devido a essas características elas podem ser consideradas inadequadas diante do advento da computação móvel, da popularização dos dispositivos móveis e da maior viabilidade proporcionada pela utilização de smartphones $e$ tablets em comparação com dispositivos antigos, como, por exemplo, os computadores de mesa.

\section{QUIRA}

O QuiRA é um aplicativo móvel baseado em Realidade Aumentada, desenvolvido com o objetivo de auxiliar no processo de ensino e aprendizagem da disciplina Química, em especial do conteúdo de Química Orgânica no contexto da Isomeria. O software permite que o usuário cadastre moléculas químicas para visualização em três dimensões (3D). A projeção dos objetos em 3D tem como referência os marcadores monocromáticos. Esses marcadores são constituídos por uma margem branca, borda preta e um identificador em seu interior, um símbolo ou uma letra, de fácil identificação no contexto computacional [10], conforme o exemplo mostrado na Figura 1.

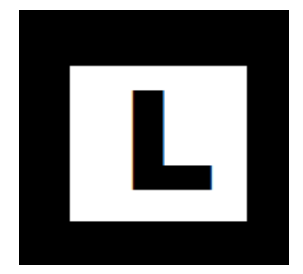

Figura 1. Marcador monocromático²

\subsection{Arquitetura do QuiRA}

A arquitetura do QuiRA (conforme exibida na Figura 2) possui os componentes ActCadObjeto, ActEdtObjeto e ActListaObjetos para as operações de inclusão, edição, listagem e exclusão das moléculas em uma tabela denominada Objeto do banco de dados. Quando o dispositivo de câmera é selecionado dentro do aplicativo, a biblioteca ARToolKit captura a imagem obtida pela câmera e envia para o componente ARWrapperObjeto. Este componente: (i) identifica o marcador presente na imagem recebida e (ii) tem acesso aos objetos cadastrados no banco de dados. Quando ARWrapperObjeto reconhece que o marcador rastreado está associado a um objeto cadastrado no banco de dados, desenha na tela do dispositivo móvel o objeto em três dimensões sobre ao marcador.

A seguir se encontram detalhadas as tecnologias e recursos mais importantes utilizados pelos componentes da arquitetura do QuiRA.

${ }^{2}$ Este marcador poderá ser utilizado posteriormente para testar o aplicativo 
QuiRA: An Augmented Reality Application to Support Chemistry Learning

\subsection{ARToolKit}

A realidade aumentada foi implementada na aplicação por meio da biblioteca Augmented Reality Toolkit (ARToolKit), uma biblioteca de visão computacional que fornece a funcionalidade de rastreamento necessária para criar aplicativos de realidade aumentada [11]. O uso do ARToolKit requer o relacionamento entre componentes escritos em linguagem de programação Java e funções em $\mathrm{C}++$. Para tanto, fez-se uso da estrutura fava Native Interface (JNI) que permite essa comunicação [12] e do Android Native Development Kit (NDK), ferramenta do Android que fornece componentes para utilizar o JNI [13]. O JNI foi empregado no contexto do QuiRA, porque este recurso melhora o desempenho da ferramenta.

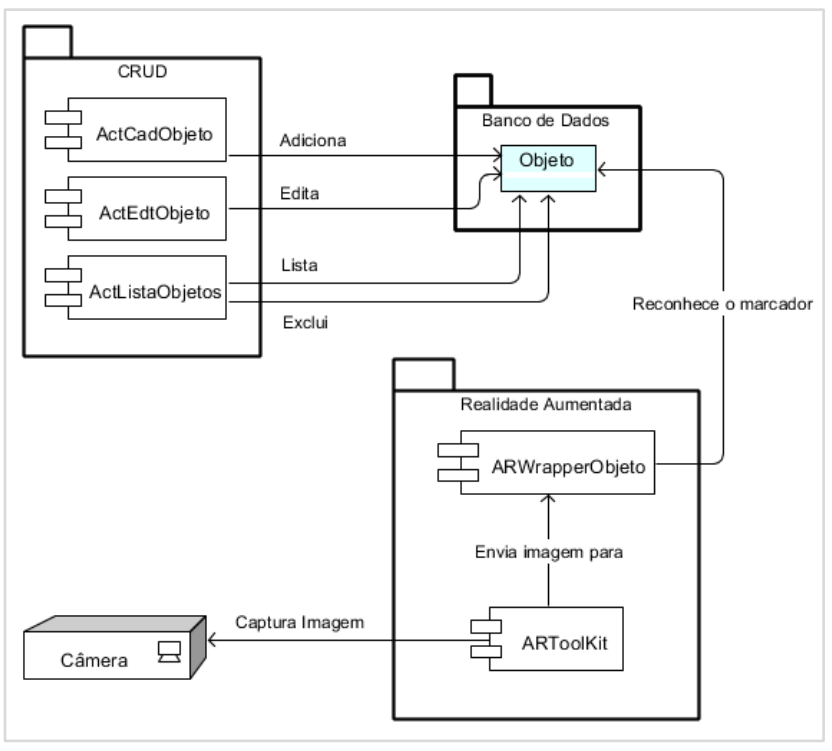

Figura 2. Arquitetura do QuiRA

O ARToolKit utiliza dois arquivos para a projeção dos objetos. O primeiro é a digitalização do marcador monocromático exportada para um arquivo no formato .patt. Para essa digitalização, a própria biblioteca fornece a aplicação Mk_Patt. O segundo arquivo é o objeto em três dimensões que deve ser exportado para o formato .obj. O arquivo .obj (OBJ) pode ser gerado através de softwares de desenho gráfico, como o Blender ${ }^{3}$, por exemplo.

\subsection{Compostos}

Para a utilização no QuiRA foram produzidos treze compostos químicos através do software Blender exportando-os para o formato .obj. Os compostos foram ácido etanoico, metanoato de metila, etanol, metoximetano, propanal,

\footnotetext{
${ }^{3}$ https://www.blender.org/
}

WebMedia'2018: Workshops e Pôsteres, Salvador, Brasil

propanona, ácido propanoico, etanoato de metila, butan-1-ol, etoxietano, but-2-en-1-ol, butan-2-ona e butanal. Na figura 3, observa-se o composto Propanal gerado.

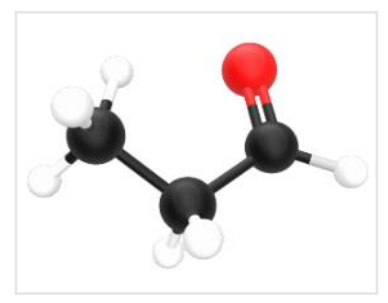

Figura 3. Propanal

\subsection{Implementação}

O processo de desenvolvimento do software envolveu: (a) a definição do banco de dados para armazenamento das moléculas e sua implementação; (b) o desenvolvimento da interface para cadastro; (c) e gerenciamento das moléculas e expansões nas classes de realidade aumentada.

Para o armazenamento das moléculas cadastradas no aplicativo, foi projetado um banco de dados no SQLite, biblioteca que implementa um banco de dados SQL e que é nativa do Android. No SQLite foi definida a tabela denominada Objeto, cujos atributos são mostrados na Tabela 1 .

Para o desenvolvimento da interface do aplicativo, definiu-se uma tela inicial com um botão de câmera para acessar a tela de visualização dos objetos 3D e com um menu com as opções de gerenciamento de objetos, de acesso ao Sobre do aplicativo e ao Manual do aplicativo. A tela de Cadastro de objeto permite a inserção do nome, descrição, arquivo .patt, arquivo .obj, imagem do objeto e imagem do marcador. Uma tela de listagem foi disponibilizada para selecionar objetos. Essa seleção permite visualizar o cadastro de um objeto. Além da visualização, um objeto pode ser modificado e excluído.

Table 1: Atributos da tabela Objeto

\begin{tabular}{cc}
\hline Atributo & Armazena \\
\hline CÓDIGO & Código identificador \\
NOME & Nome da molécula \\
DESCRICAO & Observação sobre a molécula \\
& Caminho do arquivo .patt gerado através \\
PATT & do marcador que será referência para a \\
& projeção da molécula \\
OBJ & Caminho do arquivo .obj da molécula que \\
sMAGEM & Caminho renderizado na tela \\
MARCADORem da molécula & Caminho da imagem do marcador \\
\hline
\end{tabular}

Cada tela que pode ser visualizada pelo usuário foi definida em código com uma Activity, como mostrado na Figura 4. É possível notar que a partir da tela Início é possível atingir as demais e que a tela de listagem se torna o meio através da qual 
são utilizadas as telas de inclusão, edição e edição de objetos correspondentes a moléculas.

A Realidade Aumentada também foi inserida no aplicativo amparada por uma Activity. Essa Activity efetua comunicação com o banco de dados para recuperar os caminhos dos arquivos .patt e .obj gravados através da tela de Cadastro.

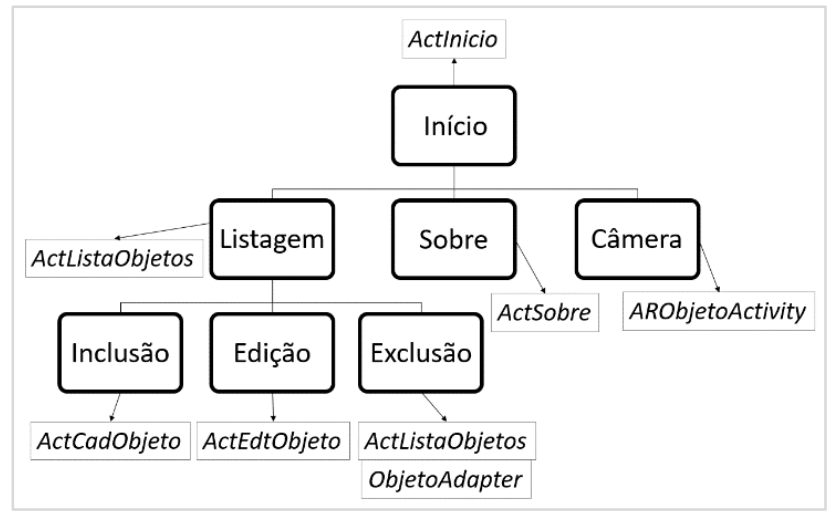

Figura 4. Telas e suas respectivas Activities

\section{UTILIZAÇÃO DO QUIRA}

O aplicativo QuiRA pode ser utilizado através da sua instalação em um dispositivo móvel com sistema operacional Android. Os materiais necessários para a sua instalação e utilização estão disponíveis no endereço bit.ly/2w8EQWA. O endereço bit.ly/2nQP8qF contém um vídeo que demonstra esse processo.

Através do endereço bit.ly/2w8EQWA citado, pode-se efetuar: (i) o download do arquivo instalador do aplicativo, (ii) obter os arquivos para cadastro da molécula química Butanal, (iii) acessar o README para obter passos rápidos para a utilização do QuiRA, (iv) visualizar o manual que ampara o processo de utilização do aplicativo de uma forma mais completa, desde a criação de marcadores até a visualização de objeto de 3D, e (v) adquirir o Digitalizador de Marcadores Mk_Patt.

Após a instalação do QuiRA, a sua tela inicial (Figura 5a) e o seu menu podem ser acessados. Com o cadastro da molécula Butanal (Figura 5b), esta pode ser visualizada através do módulo de câmera da aplicação quando a câmera do dispositivo é direcionada ao marcador relacionado. Para este caso foi cadastrado o marcador com a letra L. A medida que novas moléculas são cadastradas na aplicação, elas são listadas como na Figura 6a. O cadastro de cada molécula pode ser acessado individualmente, o que possibilita sua visualização completa e sua edição (Figura 6b). A molécula pode ser excluída da listagem por meio do botão de lixeira. Todas as moléculas cadastradas serão carregadas no momento que o módulo de câmera for acionado. Com isso elas poderão ser visualizadas em três dimensões conforme mostrado na Figura 7. E a visualização do objeto em 3D atende ao movimento do marcador.

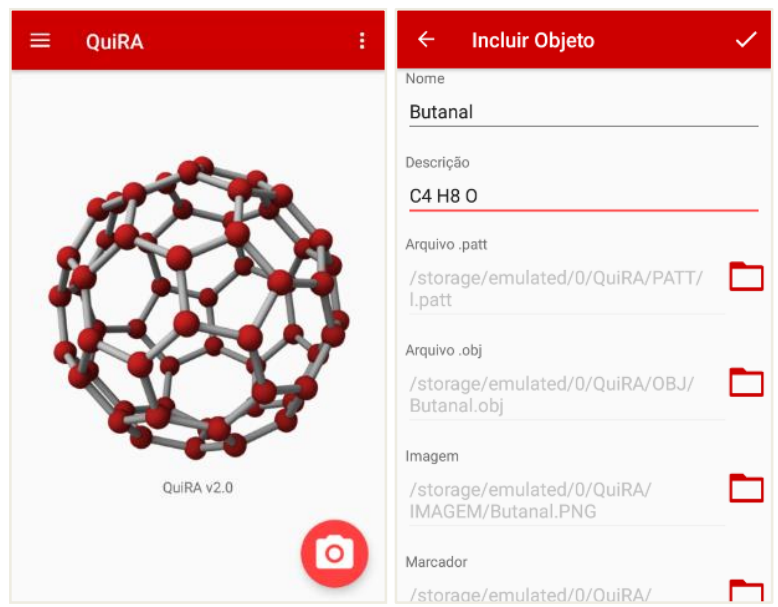

Figura 5. Telas Inicial e de Cadastro

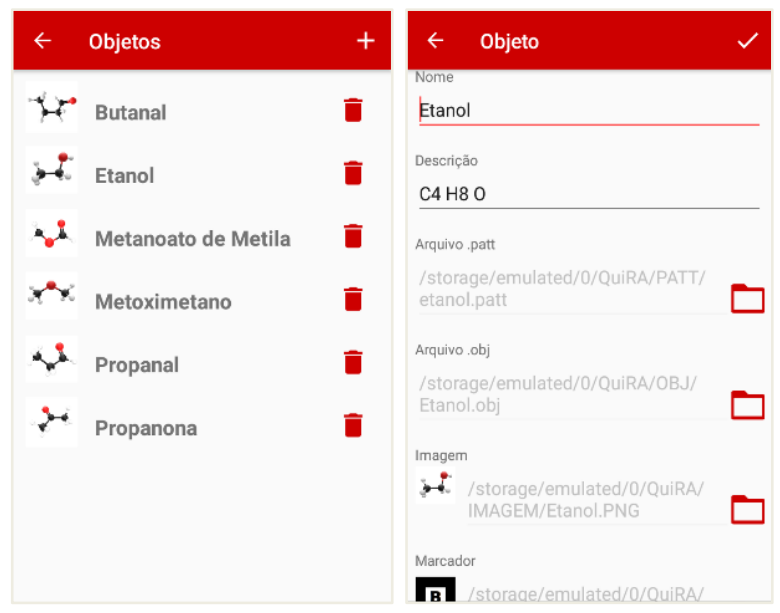

Figura 6. Telas de Listagem e de Edição
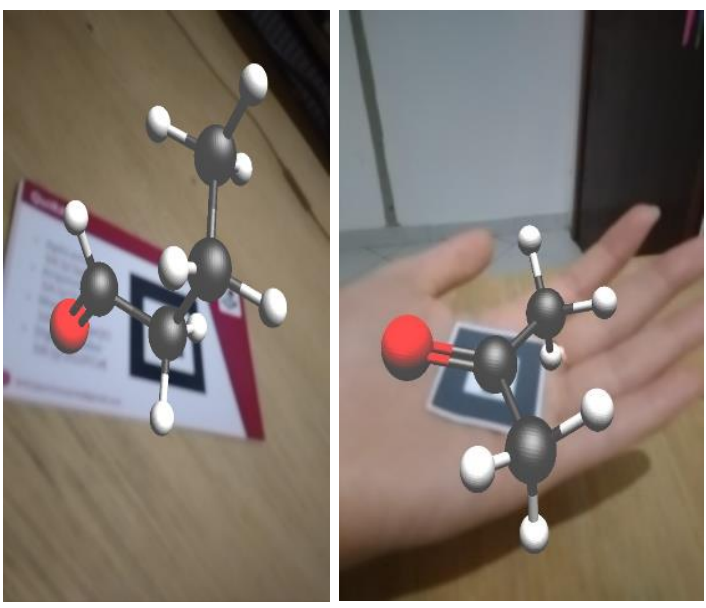

Figura 7. Visualização das moléculas Butanal e Propanona 
QuiRA: An Augmented Reality Application to Support Chemistry Learning

\section{CONCLUSÕES}

A Realidade Aumentada pode trazer uma experiência diferenciada ao usuário e o QuiRA permite que essa tecnologia seja trazida para mais perto do usuário em um alto nível de abstração. No aplicativo é possível ter a vivência com a realidade aumentada sem a necessidade de lidar com programação de códigos, tornando o contato com a RA mais acessível. Dessa forma, o professor pode utilizá-lo em sala de aula como incentivo para os alunos, já que possibilita que conteúdos intangíveis e de difícil aplicação prática, possam ser visualizados através de dispositivos que já fazem parte de seu cotidiano. O aplicativo permite que o usuário efetue o cadastro das moléculas, associando sua representação tridimensional a um marcador monocromático, a partir de arquivos armazenados no dispositivo móvel. Depois, as moléculas podem ser vistas instantaneamente através da câmera do dispositivo dentro do aplicativo, quando esta é direcionada aos marcadores cadastrados. Um trabalho futuro inclui o desenvolvimento, a partir do QuiRA, de aplicativos que amparem outras disciplinas, a fim de colaborar com uma aprendizagem mais lúdica. Outra possibilidade é desenvolver aplicativos direcionados a visualização de objetos de outras áreas, como a Arquitetura. Diante disso, o QuiRA é um projeto com pretensão de evolução e que ainda está em desenvolvimento.

Além de abordar outras áreas de ensino, também existe a intenção de avaliar o QuiRA em um experimento. Tal experimento será realizado com o objetivo de coletar opiniões de professores e alunos. Posteriormente, essas opiniões serão utilizadas para melhorar o QuiRA tanto em relação à sua usabilidade quanto, possivelmente, à sua arquitetura $\mathrm{e}$ codificação. Por isso, por enquanto, a sua licença permanece fechada, mas a intenção é que o seu código fonte seja liberado.

\section{REFERÊNCIAS}

[1] O. H. A. Araújo, L. T. F. Ribeiro e M. N. S Pinheiro. Tecnologias móveis nos processos de ensino e de aprendizagem: mobilidade docente?. Revista IberoAmericana de Estudos em Educação, v. 11, n. 1, p. 95-110, 2016, in press. Disponível em: <https://periodicos.fclar.unesp.br/iberoamericana/article/view/8087> Acesso em 10 Maio 2018

[2] R. Azuma. Recent advances in augmented reality. In v .21, n., editor, IEEE Computer Graphics and Applications, pages 34-47, in press. 2001. Disponível em: <http://www.cs.unc.edu/ azuma/cga2001.pdf> Acesso em 29 Maio 2018

[3] L. Araujo, E. Xavier, P. Vasconcelos, M. Machado e T. Tavares (2017). DoctorBio: Um estudo de caso sobre a utilização de uecursos de realidade aumentada no ensino de ciências biológicas. In: Anais do Workshop de Informática na Escola, in press. 2017. p. 294. Disponível em: <http://www.brie.org/pub/index.php/wie/article/view/7247/5045> Acesso em 10 Maio 2018

[4] A. L. Marques et al (2008). A importância de aulas práticas no ensino de química para melhor compreensão e abstração de conceitos químicos. In: Encontro Nacional de Ensino de Química, 14., 2008, Paraná. Anais... Paraná: UFPR, 2008, in press. Disponível em <http://www.quimica.ufpr.br/eduquim/eneq2008/resumos/R0727-1.pdf> Acesso em 10 Maio 2018.

[5] ARAÚJO, M. A (2017). A Realidade Aumentada no contexto educacional como mecanismo alternativo para visualização de Isômeros na Química Orgânica. Monografia (Graduação em Sistemas de Informação). Instituto Federal da Bahia, Vitória da Conquista, 2017.
WebMedia'2018: Workshops e Pôsteres, Salvador, Brasil

[6] K. K. C. Cunha, M. P. Guimarães. Desenvolvimento de uma Interface Gráfica para o Artoolkit com Aplicação na Área Educacional. In: WRVA'07 Workshop de Realidade Virtual e Aumentada, Itumbiara, 2007, p. 1-4, in press. Disponível em <http://www.lbd.dcc.ufmg.br/colecoes/wrva/2007/0030.pdf> Acesso em 10 Fev 2018.

[7] PORTAL DA INDUSTRIA. Alunos do SENAI usam aplicativos de realidade aumentada para aprender de forma divertida e inovadora. 2016. Disponível em

<http://www.portaldaindustria.com.br/agenciacni/noticias/2016/08/alunosdo-senai-usam-aplicativos-de-realidade-aumentada-para-aprender-de-formadivertida-e-inovadora/> Acesso em $10 \mathrm{Fev} 2018$.

[8] E. J. Hernandez-Leak. Construction of learning objects with Augmented Reality: An experience in secondary education. In: Learning Technologies (LACLO), 2017 Twelfth Latin American Conference on. IEEE, 2017. p. 1-7, in press. Disponível em <https://ieeexplore.ieee.org/document/8120948/authors> Acesso em 12 Maio 2018 .

[9] P. Maier, M. Tönnis, G. Klinker. Augmented Reality for teaching spatial relations. In: Conference of the International Journal of Arts \& Sciences, Toronto, 2009, in press. Disponível em < https://pdfs.semanticscholar.org/6813/b691bc09e0172051b2e77c379d74b93014 9c.pdf > Acesso em 10 Fev 2018.

[10] ANDROID PRO. Android SDK. 2017. Disponível em $<$ https://www.androidpro.com.br/blog/android-studio/android-sdk/> Acesso em 29 Maio 2018.

[11] GITHUB. android_about.md. 2016. Disponível em <https://github.com/artoolkit/artoolkitdocs/blob/master/4_Android/android_about.md> Acesso em 12 Maio 2018.

[12] GITHUB. android_developing.md. 2016. Disponível em <https://github.com/artoolkit/artoolkitdocs/blob/master/4_Android/android_developing.md> Acesso em 12 Maio 2018.

[13] ANDROID. Primeiros passos com o NDK. Disponível em <https://developer.android.com/ndk/guides/index.html?hl=pt-br> Acesso em 15 Ago 2017. 